\title{
Trending Breakthroughs in the Advances of Detection of Oral Premalignant and Malignant Lesions - A Review
}

\author{
Rakhi Chandak ${ }^{1}$, Manoj Chandak², Pranali Thakare ${ }^{3}$, Ramhari Sathawane ${ }^{4}$, \\ Swapnil Mohod ${ }^{5}$ Runal Bansod ${ }^{6}$, Pranada Deshmukh7 ${ }^{7}$ Zareesh Akhtar ${ }^{8}$ \\ 1, 3, 4, 6, 7, 8 Department of Oral Medicine \& Radiology, SDKS Dental College, Nagpur, Maharashtra, India, \\ ${ }^{2}$ Department of Endodontics and Conservative Dentistry, SPDC, DMIMSU, Sawangi, Dental College \& \\ Hospital, Wardha, Maharashtra, India. ${ }^{5}$ Department of Oral Medicine \& Radiology, SPDC, DMIMSU, \\ Sawangi, Dental College \& Hospital, Wardha, Maharashtra, India.
}

\section{ABSTRACT}

Oral cancer is the sixth most common malignant tumour, and it is the leading cause of morbidity and mortality due to its capacity to spread and invade. Oral cancer occurs at a different rate in different areas of the world, ranging from 2 to 10 per 100,000 people each year. Oral cancer is prevalent in South Asian nations such as Sri Lanka, India, Pakistan, and Bangladesh. In India, the frequency is 7-17 per 100,000 people each year, with 75,000 - 80,000 new cases per year. Identifying oral cancer in its early stages has a significant impact on survival rates when compared to detecting it later. Despite this, almost half of all diagnosed patients die within five years. A variety of well-established cancer screening programmes have been demonstrated to lower the patient morbidity and mortality dramatically. Regular check-ups, which include a thorough inspection of the whole mouth, are critical for detecting malignant and pre-cancerous problems early on. Unfortunately, early detection of oral precancerous and cancerous lesions has proved difficult due to the lesions' asymptomatic nature, doctors' casual approach to benign lesions, and the fact that 50 $\%$ of patients had regional or distant metastases at the time of diagnosis.

Oral cancer is one of the most common cancers that leads to defacement and death. Despite recent advancements in therapeutic modalities, the prognosis has not improved. Patient's mortality rates are positively associated with the point of presentation, with $60 \%$ of people diagnosed with late-stage illness. Early diagnosis is important for oral cancer patient's survival rate, as it decreases morbidity and mortality. According to the World Health Organization, the bulk of oral cancer patients are diagnosed late in the disease's progression, with a mediocre 5 - year survival rate of $50 \%$. As a result, careful treatment of oral cancer necessitates early diagnosis and intervention. Surgical biopsy is the gold standard for medical purposes, but it requires clinical assistance.

Other screening methods that are simple to use, non-invasive, and expensive are the norms for any test to be accepted as a histopathology choice. The older cancer diagnosis modalities took longer, had more inter-observer bias, and were less descriptive. A standard oral examination with digital palpation is used in traditional techniques of screening for oral possibly malignant illnesses and oral cancers. Conventional inspection has been shown to be a poor discriminator of oral mucosal lesions. A variety of visual aids have been developed to help clinicians spot anomalies in the oral mucosa and in recentyears, scientific and clinical developments have aided in the early detection and treatment of this disease. This review reflects on some of the older diagnostic modalities and screening methods for oral cancer diagnosis, as well as some of the recent more sophisticated techniques.

\section{KEY WORDS}

Diagnostic Aids, Oral Cancer, Premalignant Lesions
Corresponding Author: Dr. Rakhi Chandak Dhantoli, Nagpur, Maharashtra, India. E-mail:rakhi.chandak@sdkdentalcollege.edu.in

DOI: $10.14260 /$ jemds/2021/433

How to Cite This Article:

Chandak $R$, Chandak $M$, Thakare $P$, et al. Trending breakthroughs in the advances of detection of oral premalignant and malignant lesions - a review. J Evolution Med Dent Sci 2021;10(28):2122-2127, DOI: 10.14260/jemds/2021/433

Submission 27-02-2021,

Peer Review 11-05-2021,

Acceptance 19-05-2021,

Published 12-07-2021.

Copyright (C) 2021 Rakhi Chandak et al. This is an open access article distributed under Creative Commons Attribution License [Attribution 4.0 International (CC BY 4.0)] 


\section{BACKGROUND}

Oral cancer, also known as malignant neoplasm of the lip or malignancy of the oral cavity, is a significant health issue that affects people all over the world. Oral cancer is the most prevalent cancer in the world, ranking sixth to eighth, with over 300,000 new cases identified each year. Oral cancer is referred to as "Oral Potentially Malignant Disorders" (OPMD) by the World Health Organization. ${ }^{1}$

Squamous cell carcinomas account for $90 \%$ of oral cancers, with nicotine and alcohol use accounting for more than $80 \%$ of cases. Precancerous lesions such as leucoplakia cause 17 percent to 35 percent of oral squamous cell carcinomas (OSCC). ${ }^{2}$ The mouth's floor, tongue, gingiva, buccal mucosa, and lips are the most popular places for oral cancer. Different gender and age classes have different death rates and annual occurrence. ${ }^{3}$

According to the World Health Organization, the bulk of oral cancer patients are diagnosed late in the disease's progression, with a mediocre 5 - year survival rate of $50 \%$. As a result, careful treatment of oral cancer necessitates early diagnosis and intervention. ${ }^{4}$

\section{DIAGNOSTIC AIDS FOR DETECTION OF ORAL CANCER}

\section{Lugol's Iodine}

It is also known as Lugol's solution and is a form of iodine. It was invented in 1829 by Jean Lugol, a French chemist. It consists of 5 parts iodine, 10 parts potassium iodide, and 85 parts (distilled) water. Iodine positive state occurs when it stains heavily with iodine; iodine negative state occurs when it does not take stain.

Composition $-10 \mathrm{~g}$ potassium iodide $+5 \mathrm{~g}$ iodine (I2) + Distilled water $100 \mathrm{ml}$. Iodine staining is brown and black in the presence of glycogen. When there is a better light source, it is easier for health care providers to spot anomalies.

\section{Advantages}

1. False negative rates are poor.

2. Findings are available right away.

3. The odds of a follow-up are higher.

4. May be used to detect non-keratinized stratified squamous epithelium.

5. Easy to follow protocol

6. The treatment may be performed by a variety of health care providers.

7. Low start up and maintenance costs.

\section{Shortcomings}

1. Irritates the usual epithelium, causing damage.

2. There is a higher risk of an allergic response to iodine, which causes stomach pain, heartburn, and nausea

3. May sometimes cause shock.

4. It is not recommended for postmenopausal women. ${ }^{5}$

\section{Vital Staining}

The colour toluidine blue is used to diagnose dysplasia and oral squamous cell carcinoma. Toluidine blue is a critical stain that is acidophilic and metachromatic. Anaplastic and dysplastic cells, which have more nucleic acids than normal tissues, are treated with toluidine blue in vivo. Malignant epithelial intracellular canals are broader than regular epithelial canals, allowing the dye to penetrate deeper. It aids in the early identification, diagnosis, and control of surgical parts, as well as the detection of biopsy areas. ${ }^{6}$

When assessing the biologic capacity of possibly malignant oral lesions, experienced physicians should use TB as a complement to clinical evaluation. TB - stained clear lesions with high-risk molecular patterns preferentially predicted risk and result in cases where microscopic evidence of dysplasia was missing, according to a new study by Moyer.

Siar compared chemiluminescence to $1 \%$ tolonium chloride mouth rinse in 2005 to see how effective it was in identifying oral cancer and possibly malignant lesions. Chemiluminescence had an accuracy rate of 80.6 percent, while tolonium chloride mouth rinses had an accuracy rate of 64.5 percent. Siar tested chemiluminescence against a mouth rinse containing $1 \%$ tolonium chloride to see how effective it was in identifying oral cancer and potentially malignant lesions in 2005. Chemiluminescence was 80.6 percent accurate, while mouth rinses containing tolonium chloride were just 64.5 percent accurate.

\section{Interpretation of $T B$ Stain}

- A dark blue stain is regarded as a good sign of malignant lesion.

- Light blue preservation is thought to be a sign of premalignant lesions.

- It is called detrimental because there is no stain accumulation in the lesion.

\section{Advantages \\ 1. Marks the location of the biopsy. \\ 2. Affordability \\ 3. Non-intrusive \\ 4. Pathology accuracy to 100 percent}

\section{Disadvantages}

1. The TB stain will last in the mouth for 4 to 6 hours.

2. Specificity: it is just a scanning tool. ${ }^{5}$

\section{LAB-on-Chip (Microfluidics)}

Lab-on-a-chip (LOC) technology adapts, miniaturises, incorporates, and automates analytical laboratory procedures into a single computer (micro-total-analysis-systems). It includes biotechnology and chemistry capabilities, which have revolutionised health care diagnostics. Oral fluid samples are used in the cancer diagnosis and analysis procedure. During dental appointments, a microfluidic kit based on an affordable, disposable microfluidic tape of pre-loaded, freeze dried reagents is used in combination with a portable instrument to facilitate early diagnostics and screening.

Microfluidics is ideally suited to research living cells in a biologically important environment with diameters of a few micrometres. A saliva sample from a patient with oral precancer or cancer, for example, may be analysed on a lab-on-achip using microfluidics technology. 
The membrane related proteins serve as the foundation for pathology diagnosis. For cancer typing and staging, the calculated profile will be compared to the archived gene profiles. Microfluidic digital PCR, microfluidic single - cell RT PCR, microarray-dependent miRNA profiling, digital microfluidic method for oestrogen detection, and microvesicle - isolation chip for detecting genetic mutations were some of the techniques based on the microfluidics principle. Microfluidics for tumour cell migration allows researchers to study tumour cell migration and deformation through microgaps while keeping the cell membrane as a novel drug target. Isolation of circulating tumour cells (CTCs) is done using microfluidic technologies. Immunoaffinity, immunomagnetic, and electrokinetic concepts are used in the CTC chips based on this microfluidics.

Oral cancer screening and diagnosis have been investigated using lab-on-a-chip / microfluidics instruments. Microfluidics co-culture techniques using 3D spheroids aid in the study of tumour microenvironment, cell proliferation, cancer cell progression, and signalling between cancer cells and mesenchymal cells. Drug testing and targeting have been made possible thanks to these co-culture microfluidic techniques.

Other microelectromechanical systems (MEMS) used to isolate high purity cancer cells include immunomagnetic separation, hydrodynamic focusing and dielectrophoresis. Integrated barcode chips, nanobiochips, single-cell microassays, label-free detection chips, cell culture microarrays, and droplet - based drug screening microfluidics are only a few of the high through put MEMS - based technologies that have been used in cancers of the breast, liver, kidney, ovary and among others. ${ }^{7}$

\section{THE LOC'S OPERATION}

The LOC consists of a microfluidic system for mixing and reaction, as well as a sensor system for identification and quantification. The analysis will be done within the appointment time period, thanks to the use of a handheld computer. Colorimetric detection using optical absorption is the ideal alternative to fluorescence and electrochemical detectors. This system is capable of performing experiments on bodily fluids.

The LOC includes a microfluidic system as well as a detector system. The reactants have eight inlets, each with a different inlet for the sample to be analysed. The sample is mixed with the right number of reactants (enzymes catalysing biological reactions). Mixers are useful in a variety of biological and chemical applications because they increase mixing efficiency and allow for faster homogenization.

Since immunoassays need a wide variety of microfluidic components and devices, the microfluidic technologies designed for pathogen detection can be extended and generalised to the more complex task of cancer screening and diagnosis. Simply put, an automated immunoassay with a single cancer marker or a panel of cancer markers with a cancer - specific gene transcription or expression profile may be introduced into a credit card sized microfluidic cassette for point-of-care cancer screening.

\section{LOC in the Diagnosis of Oral Cancer}

Genetic changes in cancer cells can trigger changes in gene expression patterns that can be observed well before the cancer phenotype manifests. In contrast to normal mucosa, cancer cells have variations that can be used as biomarkers. p53, cyclin D1, and the epidermal growth factor receptor gene are just a few of the candidate genes linked to OSCC tumour formation.

According to a microarray analysis of different tumour forms, global expression profiling distinguishes tumour cells from normal cells. These gene signatures can now be integrated into microfluidic LOC structures thanks to the advent of high density microarrays and advances in bioinformatics. According to science, OSCC has been shown to down regulate or up regulate a variety of genes.

Because of the introduction of high density microarrays and advancements in bioinformatics, these gene signatures can now be inserted into microfluidic LOC frameworks.

To isolate cancer cells from the sample, magnetic beads coated with anti - EpCAM antibody are used. EpCAM is a protein found on the surface of cancerous epithelial cells that is abnormally expressed. It is possible to easily identify and count the cancer cells that have been separated. On differentiated cancer cells, the isolated mRNA is then subjected to a thermal and / or chemical lysis stage.

Reverse transcription polymerase chain reaction, linear amplification, or a bio - barcode technique may both be used for multiplex mRNA amplification. To classify the type of cancer, the transcription profile of isolated sample cancer cells is compared to cancer signature profiles archived in a database using established statistical principles.

\section{Potential Uses}

1. Drug development and manufacturing mechanisms, as well as treatment effectiveness and resistance.

2. Neurotransmitter identification, cell culture and growth, disease progression, and occurrence.

3. Identification and classification of microorganisms (HIV, malaria, tuberculosis, Escherichia coli, salmonella, shigella), viruses and proteins.

4. Detects toxins, bombs, and nitroaromatics in the atmosphere.

5. Counts of sperm, motility, and urine examination

6. Diagnosis of an acute myocardial infarction.

\section{Advantages}

1. A technique that is minimally invasive that uses a small sample size.

2. Method that is fully automated.

3. Fast response times.

4. Reproducibility, consumption, and accuracy of the reagents are all diminished.

5. The risk of being exposed to dangerous contaminants is reduced.

6. There is a reduced risk of sample leakage.

7. Convenient disposal.

8. Portability in minor sizes.

9. There is no need of electricity infrastructure.

10. Human error is eliminated.

11. Advanced method of analysis. 
12. Operational capability for staff with limited training

\section{Disadvantages}

1. Inadequate fluid flow interfaces;

2. Difficult production process

\section{Future Prospects}

For the sake of bettering both the climate and global wellbeing, Lab on Chip technology has the potential to be a critical factor. Saliva is used as a screening opportunity for study and commercialization to better explain genomics, proteomics, and transcriptomics. Saliva will be used as a medical screening for a single illness in the future for a whole population. Oral cancer Lab on Chip research, a new area in nanotechnology and microbiology, allows for faster detection and better prognosis. $^{8}$

\section{Contact Endoscopy (CE)}

It allows for rapid diagnosis and has the unique ability to demonstrate confirmed cellular architecture in vivo. CE can analyse the mucosa's cellular architecture and vascular pattern in real time, and it can be replicated as many times as possible. It can also scan vast areas easily. This function is critical in determining the best location for a biopsy. CE prevents tissue damage and modifications that can occur during biopsy. CE also prevents tissue alterations that can occur during histopathological analysis. It is not recommended as a replacement biopsy at this time. It is useful for detecting the biopsy region and is a non-invasive procedure with high precision, accuracy, and ease of use for evaluating oral mucosal lesions. ${ }^{9}$

\section{Advantages}

1. Contact endoscopy is non-invasive, repeatable, fast, and produces results quickly.

2. It will include more data, allowing for a more thorough assessment of larger surface areas.

3. CE can help to prevent tissue injury and cellular architecture damage.

4. It aids in the detection of the biopsy site and the identification of benign and malignant mucosal lesions.

\section{Disadvantages}

Because of the following considerations, contact endoscopy was used to determine the mucosal epithelium's most superficial cell layer.

1. Methylene blue just stains the top few layers of the skin.

2. The scope's focal distance is inadequate.

3. Because of optical artefact, evaluating submucosal lesions at deeper cell layers is challenging. ${ }^{10}$

\section{Optical Coherence Tomography (OCT)}

Low - power infrared light between 750 and $1300 \mathrm{~nm}$ is used in this modality. Images are produced in OCT by measuring the echo time delay and measuring the strength of backscattered light. Scattering occurs as light interacts with the tissue surface. The probe used in optical coherence tomography (OCT) imaging has a penetration depth of 1 to $3 \mathrm{~mm}$.
OCT may analyse and assess architectural changes in the keratin cell layer, lamina propria, epithelial layer, basement membrane, rete pegs of the oral mucosa, and neoplastic transition. OCT has long been used in ophthalmology for in situ "optical biopsy" of the retina, as well as in dermatology for skin tumour examination and inflammatory disorders. Although OCT can assess lesions for neoplastic changes by assessing epithelial layer thickness, basement membrane integrity, and changes in the lamina propria, it cannot score OPMDs due to a lack of cellular information.

Early research showed that, while OCT imaging had 93 percent sensitivity and 97 percent accuracy for diagnosing OSCCs as compared to histology in one study, it had little ability to differentiate between various oral mucosal abnormalities with another. Ex vivo biopsies have a sensitivity and precision of 85 percent and 78 percent, respectively, for the identification of OPMDs, according to recent reports. Tsai et al. looked at OCT profiles for OSCC margin delineation and suggested that this information could be used to construct an OSCC detection and delineation algorithm. More study is required on the potential use of OCT to improve and classify excisional margins during surgical management of OPMDs and OSCCs. ${ }^{11}$

\section{Advantages}

1. Optical coherence tomography provides in situ imaging without the need to excise a specimen.

2. Offers 3D views in real time.

3. It's a small and portable unit.

\section{Disadvantages}

1. Blood obstructs clear photographs

2. Inability to penetrate. 6

\section{Microarrays}

New technology, such as DNA microarrays and DNA chips, have been created that provides hundreds of thousands of genetic details in a shorter period of time than traditional PCR techniques. Microarrays are useful for classifying tumour subtypes because molecular information can be combined and collected from a group of samples to find similar patterns.

Microarrays are used in conjunction with other screening tools to add additional detail about the tumour specimen by looking at thousands of genes at the same time. Microarrays are used to classify tumour samples into taxonomic groups, as well as diagnostic and therapeutic markers. New subtypes are linked to patient success. ${ }^{12}$

\section{Advantages}

1. A qualitative investigation of mRNA

2. Gene identification in a single step.

\section{Disadvantages}

1. Extensive and long-term labour.

2. Needs the preservation of DNA

3. Hybridization by cross pollination. ${ }^{6}$

\section{Nano Diagnostics}

It is a cost-effective modality that uses nanotechnology for earlier disease diagnosis and improved sensitivity in clinical 
diagnostics. Nanotechnologies for diagnostic applications are beneficial in meeting the clinical laboratory's stringent requirements for precision and cost effectiveness. Gold nanoparticles (AuNPs), quantum dots (QDs), and cantilevers are recent nanodiagnostic instruments that are useful for diagnostic applications due to their high photo stability, single wavelength excitation, and size tunable emission nanocrystals. Intracellular imaging, tissue imaging, tumour detection, immunohistochemistry, infectious agent detection, fluoroimmunoassays, and multiplexed diagnostics are only a few of the possible diagnostic applications of QDs. For many diagnostic uses, including intracellular imaging, nanodiagnostics offer multiplexing capabilities, improved sensitivity, and lower costs. ${ }^{13}$

\section{Advantages}

1. It is flexible, adaptive, to high speed biological tests

2. It is usable for small samples

3. In cancer treatment, it can be used for targeted therapy.

4. It is cost effective.

\section{Disadvantages}

1. Nanoparticles are harmful to people's health.

2. Legal / ethical issues 6 .

\section{Orascoptic DK}

It is a versatile product that combines a handheld LED light source with three interchangeable diagnostic tools: A transilluminating instrument, a lighted instrument with mirror, and an oral lesion screening instrument. With a penetrating, concentrated source of light, the DK Transillumination instrument aids in the imaging of dental caries and fracture teeth. The DK Lighted Mirror, which comes with a powerful LED light source to brighten up the reflection received from the mirror, aids in clearly visualising the unreachable areas inside the oral cavity. The technique is equivalent to Vizilite. The conventional oral scrutiny is done using an incandescent light. Oral lesion screening instrument, examined oral mucosa again upon rinsing with $1 \%$ acetic acid mouth rinse, and bluish white light was emitted. The 'aceto-white lesions' look brighter thus helping in better visualization of lesions. ${ }^{14}$

\section{Chemiluminescence}

For identifying, monitoring and evaluation of oral premalignant lesions, chemiluminescence is used as an additional modality to the conventional modality of oral examination. Other uses of this technology include immunoassays, liquid chromatography, and pharmaceutical analysis measurement of important enzymes, metabolites, gene probe assays and chemiluminescence imaging. Other terms for chemiluminescence used for oral mucosal lesions include Vizilite, MicroLux DL, and Vizilite Plus. The MicroLux device contains a reusable, battery powered source of light, while Vizilite Plus uses a disposable chemiluminescence light packet.

\section{Vizilite Used in Screening Procedure}

The extent of the lesion and features of the mucosal lesion are reported during a traditional examination after the oral cavity is examined by dental chair light if some was found through a standard inspection. Take a snapshot of the lesion and instruct the patient to clean the oral cavity with $30 \mathrm{~mL}$ of $1 \%$ acetic acid and wash it out after one minute. This helps to clear the glycoprotein layer and dries the oral mucosa, allowing the Vizilite capsule to be activated.

Place the capsule in the Vizilite retractor after shaking it to mix the contents. In a dimly lit setting, an oral cavity inspection may be performed. Natural epithelium appears blue, while altered epithelium appears acetowhite.

For the toluidine blue labelling system, the patient is asked to rinse the oral cavity with $10 \mathrm{ml}$ of $1 \%$ tolonium chloride and wash it after a minute, then rinse the oral cavity with $10 \mathrm{ml}$ of $1 \%$ acetic acid and wash it out after $20 \mathrm{~s}$, then photograph and report all the results.

\section{Mechanism of Action}

This technique detects metabolic and structural changes in mucosal tissues exposed to multiple types of light sources, which have different absorbance and reflectance properties by nature. Acetic acid solution is used as a cytoplasmic dehydration agent. It ostensibly destroys ions and breaks down the epithelium's glycoprotein barrier, allowing light to pass through. As chemiluminescent light is applied, the blue white light is absorbed by normal mucosal cells and reflected by cells with abnormal nuclei, such as dysplastic and neoplastic cells, giving the dysplastic and neoplastic epithelium a "white" appearance, hence the term "acetowhite."

As a result, the "acetowhite" lesions appear white, while the usual epithelium appears blue. The two compounds in the Vizilite capsule, acetyl salicylic acid and hydrogen peroxide, combine to produce steam. This energy is taken up by a fluorescent dye, which converts it into light. The colour of the fluorescent dye decides the colour of the light stick that results when the chemical solutions are mixed.

The basic theory of the reaction is that the reaction between the two chemicals provides enough energy to excite the electrons in the fluorescent dye. The electrons would then ascend to a higher energy level before plummeting and emitting light. Hydrogen peroxide oxidises the phenyl oxalate ester, forming phenol and peroxyacid ester. Phenol and a cyclic peroxy complex are formed as the unstable peroxyacid ester decomposes. When the cyclic peroxy compound decomposes, carbon dioxide is produced.

During the decomposition reaction, the energy that excites the dye is released. A difference in epithelium thickness or the inclusion of a higher density of nuclear material and mitochondrial matrix, which preferentially reflects light, could explain the observed signals. Hyperkeratinized or dysplastic lesions appear remarkably white when seen under diffuse low -energy wavelength illumination. To visualise and label suspicious oral lesions, Vizilite plus uses two instruments, toluidine blue, an acidophilic dye that selectively stains acidic tissue components including deoxyribonucleic acid and ribonucleic acid, was used in this labelling scheme. Owing to increased nuclear density, larger intracellular canals that allow dye penetration, loss of cell cohesion, and increased mitosis, the dysplastic and anaplastic cells are painted with toluidine blue, while the regular epithelium remaining unstained. Chemiluminescence is used in combination with a convectional oral mucosal examination to help in the 
identification, diagnosis, and management of oral lesions with a high risk of cancer. Other uses of this technology include immunoassays, gene probe assays, measurement of essential enzymes and metabolites, liquid chromatography, pharmaceutical testing, and chemiluminescence imaging.

\section{Advantages}

1. It is easy, healthy, and non-toxic to biological tissues;

2. It provides instant effects, has limited operator complexity, and is simple to understand.

3. Because of its single use and non-invasive existence, there are less chances of cross contamination.

\section{Disadvantages}

1. Expensive, unable to detect the biopsy location

2. The technique's accuracy, precision, and efficacy are insufficient.

3. It is used only once on each patient.

4. It is impossible to visualise the calculation effects. 15 "

Furthermore, early diagnosis would lessen mortality from oral cancer and improve the quality of life. An increased clinical suspicion and determinants of patient's delay may help accomplish earlier diagnosis by minimizing the causes for "delay. 16 " Therefore, it is also equally important to detect and control the premalignant lesions and conditions. The detection of multifocal cancerous process at an earlier stage with scrupulous diagnostic procedures for better treatment should improve survival in these patients. ${ }^{17}$

\section{CONCLUSIONS}

Oral cancer screening and diagnosis is a huge concern for both dental and medical services these days. Oral cancer screening and early diagnosis are needed in populations that are at risk of developing the disease, in order to reduce morbidity and mortality. Oral disease identification, diagnosis, and treatment can be difficult. Surgical biopsy is a gold standard diagnostic procedure, and several diagnostic procedures have been attempted to replace it. However, it is used as an adjuvant to surgical biopsy rather than as a replacement. Clinical trials should be sound and methodological when testing newer approaches, and they should be trained, compared and quantified to surgical biopsy. In the detection of tumours, highly modern methods enhance medical treatment. Continual studies would undoubtedly boost the ability to detect the illness at the earliest possible time. New developments can play a bigger role in early intervention with less impairment and a higher chance of cure.

Financial or other competing interests: None.

Disclosure forms provided by the authors are available with the full text of this article at jemds.com.

\section{REFERENCES}

[1] Carreras-Torras C, Gay-Escoda C. Techniques for early diagnosis of oral squamous cell carcinoma: systematic review. Med Oral Patol Oral Cir Bucal 2015;20(3):305-15.

[2] Fourie J. VEL scope: shedding light on its ideal application. S Afr Dent J 2018;73(2):71-7.

[3] Chaudhary R, Shah A, Shah DM, et al. Advanced diagnostic aids in detection of oral cancer. J Dent Med Res 2014;1(3):139-43.

[4] Sharma G. Diagnostic aids in detection of oral cancer: an update. World J Stomatol 2015;4(3):115-20.

[5] Reddy GS, Rao KE, Kumar KK, et al. Diagnosis of oral cancer: the past and present. J Orofac Sci 2014;6(1):10-6.

[6] Nambiar KS, Haragannavar VC, Augustine D, et al. Diagnostic aids in detection of oral precancer and cancer: past to present. International Dental \& Medical Journal of Advanced Research 2016;2(1):1-7.

[7] Ziober BL, Mauk MG, Falls EM, et al. Lab-on-a-chip for oral cancer screening and diagnosis. Head Neck 2008;30(1):111-21.

[8] Pandya D, Nagarajappa AK, Reddy S, et al. Lab-on-a-chiporal cancer diagnosis at your door step. Journal of International Oral Health 2015;7(11):122-8.

[9] Mishra AK, Nilakantan A, Sahai K, et al. Contact endoscopy of mucosal lesions of oral cavity-preliminary experience. Med J Armed Forces India 2014;70(3):257-63.

[10] Szeto C, Wehrli B, Whelan F, et al. Contact endoscopy as a novel technique in the detection and diagnosis of mucosal lesions in the head and neck: a brief review. J Oncol 2011;2011:196302.

[11] Bhatia N, Lalla Y, Vu AN, et al. Advances in optical adjunctive AIDS for visualisation and detection of oral malignant and potentially malignant lesions. Int J Dent 2013;2013:14029.

[12] Shwetha KN, Vanishri HC, Dominic A, et al. Recent advances in diagnosis of oral cancer. Journal of Dental and Orofacial Research 2016;12(1):19-21.

[13] Naikmasur VG, Sattur AP, Mutalik S, et al. Recent advances in diagnostic oral medicine. Journal of Indian Academy of Oral Medicine and Radiology 2009;21(3):99-104.

[14] Niyogi S, Priyadarshini S. Non-invasive chairside diagnostic techniques: a review. Indian Journal of Public Health Research \& Development 2019;10(11):289-93.

[15] Shashidara R, Sreeshyla HS, Sudheendra US. Chemiluminescence: a diagnostic adjunct in oral precancer and cancer: a review. J Cancer Res Ther 2014;10(3):487-91.

[16] Lohe VK, Bhowate RR, Sune RV, et al. Association of socioeconomic risk factor with patients delay in presentation of oral squamous cell carcinoma. Journal of Datta Meghe Institute of Medical Sciences University 2017;12(1):75-8.

[17] Sune RV, Indurkar AD, Bhowate RR, et al. An evaluation of field cancerization in patients with oral cancer by mirror image biopsy. Journal of Datta Meghe Institute of Medical Sciences University 2017;12(2):148-53. 